\title{
Información de salud: fuentes periodísticas y desafíos profesionales
}

\section{Health information: Media sources and professional challenges}

\author{
Marta Saavedra-Llamas; Mercedes Herrero-De-la-Fuente; Leticia Rodríguez- \\ Fernández; Carlos Jiménez-Narros
}

Cómo citar este artículo:

Saavedra-Llamas, Marta; Herrero-De-la-Fuente, Mercedes; Rodríguez-Fernández, Leticia; Jiménez-Narros, Carlos (2019). "Información de salud: fuentes periodísticas y desafíos profesionales". El profesional de la información, v. 28, n. 2, e280208.

https://doi.org/10.3145/epi.2019.mar.08

Artículo recibido el 07-11-2018 Aceptación definitiva: 05-02-2019

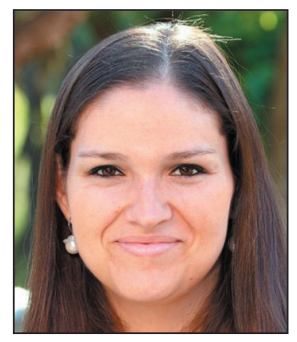

Marta Saavedra-Llamas $ه$ https://orcid.org/0000-0002-7762-9996

Universidad Nebrija

Santa Cruz de Marcenado, 27. 28015 Madrid, España

msaavedr@nebrija.es

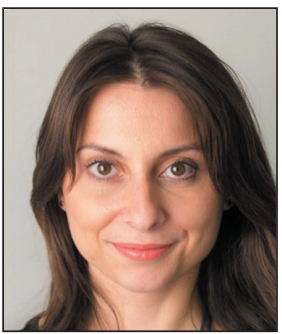

Leticia Rodríguez-Fernández https://orcid.org/0000-0002-7472-5472

Universidad Nebrija

Santa Cruz de Marcenado, 27. 28015 Madrid, España

Irodrigu@nebrija.es

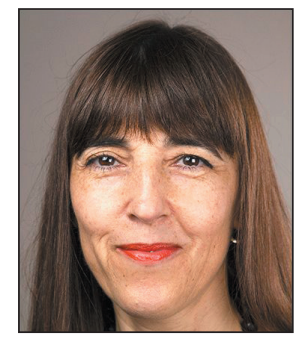

Mercedes Herrero-De-la-Fuente https://orcid.org/0000-0002-5361-9056

Universidad Nebrija

Santa Cruz de Marcenado, 27. 28015 Madrid, España

mherrero@nebrija.es

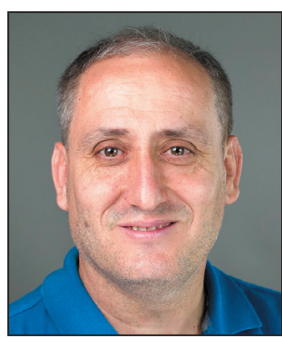

\section{Carlos Jiménez-Narros}

https://orcid.org/0000-0001-8311-0261

Universidad Nebrija

Santa Cruz de Marcenado, 27. 28015 Madrid, España

cjimenez@nebrija.es

\section{Resumen}

Los periodistas especializados en información de salud han modificado sus rutinas periodísticas por el impacto de internet y las redes sociales. La generación de estos contenidos permite conectar a investigadores, facultativos y pacientes y, en este sentido, los medios de comunicación desempeñan un papel prescriptor sobre la sociedad. Analizamos si la precariedad imperante en los medios de comunicación afecta a la información de salud y a la especialización de sus profesionales. El trabajo se fundamenta en una investigación cualitativa con entrevistas en profundidad a profesionales de la información de salud pertenecientes a 16 medios de comunicación españoles. El estudio realizado pone de relieve la prioridad de determinadas fuentes de consulta, la necesidad de activar procesos de verificación y de hacer frente a nuevos retos profesionales. 


\title{
Palabras clave
}

Información de salud; Periodismo especializado; Periodistas; Medios de comunicación; Fuentes de información; Rutinas periodísticas; Redes sociales; Medios sociales; Internet; Retos profesionales; Verificación; Investigación cualitativa.

\begin{abstract}
Journalists specialized in health information have changed their work routines because of Internet and social networks. Generating health content allows researchers, physicians and patients to be connected and, in this respect, media play a prescriber role in the society. In this paper, the prevailing precariousness in the media is analyzed in order to see if it affects health information and the specialization of its professionals. This work is based on a qualitative research, using in-depth interviews with health information journalists belonging to 16 Spanish media. The research underscores the priority of certain resources, the need to enable verification processes and to face new professional challenges. This research is part of the Aula Nebrija-MSD Spain of Communication and Health Sciences funded project.
\end{abstract}

\section{Keywords}

Health information; Specialized journalism; Journalists; Media; Information sources; Journalistic routines; Social media; Social networks; Internet; Professional challenges; Verification; Qualitative research.

\section{Información de salud en medios de comunicación}

Se trata de una especialización periodística compleja, de alto impacto social y con retos profesionales permanentes. Revuelta (2006) indica dos condiciones necesarias en la construcción de esta información especializada:

- contener una serie de valores intrínsecos como novedad, impacto social, impacto emocional o gran dimensión, entre otros;

- pasar por un circuito de información eficiente.

En este proceso de creación de contenidos informativos, el periodista especializado se convierte en protagonista por tratarse de un profesional con conocimientos en el área de la salud y por su capacidad para adaptarse a las circunstancias de su especialidad. Waisbord y Coe (2002) señalan, entre otros, estos desafíos:

- superar la tentación de informar sobre resultados prematuros que no han sido comprobados;

- evitar simplificar los resultados que ofrezcan distintas investigaciones a lo largo del tiempo;

- eludir la confusión entre publicidad y periodismo, diferenciando la información ofrecida desde los departamentos de comunicación de empresas del sector;

- superar el sensacionalismo.

Sobre este último punto sugieren los autores que la información sobre salud recoge a menudo lo que es atractivo como noticia, más que lo relevante a largo plazo.

Los cambios tecnológicos y la crisis económica global iniciada en 2008 han planteado nuevos retos para el periodista especializado en salud:

1) El primero es la pérdida de influencia de los medios tradicionales, que manejan presupuestos cada vez más reducidos y compiten con los nuevos soportes para las informaciones científicas en internet. Muestra de ello es que en 2004 el $33,3 \%$ de los diarios con mayor difusión en España tenía alguna sección dedicada a ciencia, salud o tecnología, aunque el 27\% declaró no disponer de ningún redactor especializado en esta área (Revuelta; Minelli-de-Oliveira, 2008, p. 205); mientras que en 2017 la información de salud ocupa tan solo el 4\% del contenido en medios generalistas (Infoperiodistas, 2018, p. 46). Lo mismo se observa en los resultados del análisis de Ronco-López, Peñafiel y Echegaray (2014, p. 277) sobre el Informe Quiral. Este trabajo estudiaba anualmente la presentación de información de salud en medios. Entre 1997 y 2006 el interés fue al alza y sobre los 5 diarios analizados se recogieron 88.555 textos relacionados. A partir de 2009 y justificado en la transformación experimentada en los hábitos de consumo informativo de la ciudadanía, el informe cambia su objeto de estudio y se centra en realizar monografías especializadas. Esta modificación se enmarca en la eclosión de medios digitales especializados que ha dado acceso a los usuarios a contenidos gratuitos. En 2010 se registraron cerca de 300 blogs sanitarios activos en castellano (Traver-Salcedo; Fernández-Luque, 2011, p. 8). A ellos se añaden los medios de comunicación digitales que aumentan el volumen de contenidos relacionados. El Infonómetro de Salud de 2018 muestra que el 70\% de la información sanitaria recogida en 2017 se publicó en medios de comunicación digitales, siendo además los medios generalistas los que difundieron mayor cantidad de información del área, el 62\%, (Infonómetro, 2018, pp. 48-49).

2) El segundo reto tiene que ver con las condiciones laborales del periodista de salud, que se caracterizan por la incertidumbre y la caída salarial, dentro de unos equipos de trabajo cada vez más reducidos en los que la información de salud se localiza en ocasiones en el área de Sociedad (González-Borjas, 2004). Casi la mitad de los participantes de un reciente estudio reconoce este deterioro: un $28,3 \%$ responde que en los últimos cinco años su estabilidad laboral ha disminuido algo y un $19,6 \%$ que ha disminuido mucho. En cuanto a los salarios, 
"un 31,9\% opina que han disminuido algo, mientras que para el 21,3\% han disminuido mucho" (Cortiñas-Rovira; Lazcano-Peña; Pont-Sorribes, 2015, p. 144).

Ufarte-Ruiz (2012) alerta igualmente sobre el deterioro de la calidad informativa al disponerse de menos tiempo y recursos. A nivel internacional, Bauer et al. (2013) llegan a esta misma conclusión, especialmente en Europa, EUA y Canadá. Esto ocurre en unas redacciones que han ido mermando al tiempo que se multiplica la figura del freelance y crece la tendencia a la externalización. El último Informe anual de la profesión periodística de la Asociación de Periodistas de Madrid $(A P M, 2017)$ cifra en alrededor del $25 \%$ los periodistas dentro de esta categoría, aunque estima que el dato puede estar distorsionado, porque muchos de los que ejercen como autónomos no pertenecen a asociaciones de periodistas y, por tanto, no son consultados para realizar la encuesta (APM, 2017, p. 8). Este informe señala también que

"la mayoría de ellos lleva menos de cinco años como autónomo y casi el $80 \%$ de los que trabajan de freelance se vio forzado por las circunstancias" (APM, 2017, pp. 84-86).

En este panorama han irrumpido como proveedoras de información de salud entidades, organizaciones y numerosas empresas relacionadas, cuyos gabinetes de comunicación se han beneficiado de la migración de periodistas científicos procedentes de los medios (Cortiñas-Rovira; Lazcano-Peña; Pont-Sorribes, 2015).

3) Este contexto nos conduce al tercer reto: el impacto sobre las rutinas periodísticas para unos profesionales a menudo sobrecargados de trabajo y que desempeñan labores para otras secciones distintas a la de salud. De hecho el $47,9 \%$ afirma hacerlo ocasionalmente y el 25\% de forma habitual (Cortiñas-Rovira; Lazcano-Peña; Pont-Sorribes, 2015, p. 145). La falta de tiempo incide en la elaboración de informaciones propias y dificulta la verificación de las fuentes. El informe E-health focus on (EHON) sobre la información de salud en internet (EHON, 2017), que recoge los resultados de un foro de debate entre periodistas, profesionales sanitarios y pacientes, organizado por la Asociación de Investigadores en eSalud (AIES) en noviembre de 2016, insiste en el exceso de información y la dificultad para identificar las fuentes. Los periodistas, en concreto, constatan que la multitarea merma la calidad de la información y los tiempos de trabajo cada vez más cortos impiden contrastar la misma. La mitad de los informadores participantes reconoce haber compartido contenidos en las redes sociales sin hacer más indagaciones, porque confiaban en la fuente. Cassany-Viladomat, Cortiñas-Rovira y Elduque-Busquets (2018) recogen que los periodistas científicos admiten carencias en su formación y la necesidad de ser más críticos con las fuentes e informaciones científicas.

De manera similar, el Infonómetro (2018) muestra que el $64,6 \%$ de los periodistas encuestados perciben la información de internet como imprecisa, ambigua y contradictoria, aunque supone casi la mitad de las fuentes de consulta (44,9\%): webs específicas $(20,1 \%)$, Wikipedia

$(12,9 \%)$ y Twitter (11,9\%). Si bien las nuevas herramientas digitales y las redes sociales son competencia esencial de cualquier periodista y forman parte de su rutina de trabajo, es necesario reivindicar también que el periodista de salud es el interlocutor del trabajo de los investigadores y que de su capacitación depende la comunicación pública de la ciencia, la salud y la tecnología. Su profesión

"contribuye a alejar el periodismo de un declive avivado por internet y las TIC que facilitan a los científicos comunicarse directamente con la sociedad, prescindiendo de mediadores" (Meneses-Fernández; Martín-Gutiérrez, 2015, p. 4).

Ante la sobrecarga informativa se ha de reforzar la labor interpretativa y analítica del periodista, que nunca podrá ser sustituida por la innovación tecnológica (Wellcomm, 2012).

Por otra parte, internet es una de las principales fuentes de información en el área de salud para profesionales sanitarios (Jiménez-Pernett et al., 2007) y para la sociedad: 8 de cada 10 personas acuden a "Doctor Google" para realizar consultas sobre salud (Pfizer, 2010, p. 27). En 2012 y según un estudio de Manning Selvage \& Lee (MSL Group) realizado con 1.200 pacientes, el 72\% reconocía utilizar la Red para búsquedas en el área, siendo las fuentes más consultadas los foros (42\%), Google (41\%) y Wikipedia (27\%) (Catalán-Matamoros, 2015). Igualmente se ha concluido en otras investigaciones que internet

"produce cambios en la forma de pensar y en los comportamientos de salud, principalmente en menores de 45 años" (Marín-Torres et al., 2013, p. 51).

La sobreabundancia de información en la Red en torno a la salud ha generado gran desconfianza entre los pacientes, aunque la Red sigue siendo la primera fuente de documentación, una vez se produce sintomatología. Con esa información el paciente acude a la consulta del médico a exponer su situación (Traver-Salcedo; Fernández-Luque, 2011) y se presupone que todos los participantes en el circuito de información, especialmente los periodistas, deberían ser conscientes de la responsabilidad intrínseca de los contenidos en la Red sobre el sector salud. 


\section{Objetivos y metodología}

Los objetivos del presente estudio son:

1. Observar la situación de la información de salud y de las redacciones especializadas en los medios de comunicación españoles.

2. Determinar si las fuentes periodísticas han evolucionado o se han ampliado tras la digitalización y la generalización de las redes sociales.

3. Advertir si las rutinas periodísticas se han visto modificadas y, en especial, analizar si los procesos de verificación se producen de una manera adecuada.

4. Cuestionar si la información de salud mantiene el rigor y la credibilidad suficiente o si se está viendo afectada por la situación de las redacciones y la agilidad que imponen los medios digitales y el consumidor actual.

Partimos de una investigación descriptiva basada en fuentes secundarias, que sirve para contextualizar la situación de la información especializada en salud en los medios de comunicación. Se pretende:

- explorar a través de la bibliografía científica publicada y de informes sectoriales de referencia, el proceso de construcción de la información sobre salud;

- abordar la situación de las secciones especializadas en este ámbito dentro de los medios nacionales;

- atender los cambios producidos en las rutinas profesionales tras la implantación de las nuevas tecnologías de la información y las redes sociales;

- observar si han variado el tipo de fuentes utilizadas y los procesos de verificación.

Tras este marco introductorio, el estudio se completa con una investigación cualitativa basada en entrevistas en profundidad. El objetivo era contrastar la situación actual de la información de salud con los profesionales de los medios de comunicación españoles, sobre todo en relación con las fuentes de consulta y los desafíos del periodista especializado.

Para ello se elaboró un cuestionario semiestructurado dividido en tres partes:

- fuentes de consulta de la información de salud y fiabilidad de los datos digitales;

- procesos de verificación de la información obtenida;

- retos y desafíos del periodismo de salud. Problemáticas derivadas de la digitalización.

En una primera fase se elaboró un mapa con medios de comunicación nacionales representativos de:

- prensa impresa y digital, nacional y regional;

- radio;

- televisión;

- agencias.

Se conformó una base de datos de 44 medios manejando criterios como ámbito nacional y cifras de audiencia, sobre todo para televisión y radio. En prensa escrita se seleccionaron las grandes cabeceras y diarios exclusivamente online. Se identificaron sus responsables en salud o en ciencia y sociedad ya que se constató, en una fase temprana del estudio, que esta especialización estaba perdiendo peso en las redacciones, bien por ajustes económicos o por quedar absorbida por las macro especializaciones señaladas.

Tras un primer contacto con los periodistas de estos medios, se concertaron 16 entrevistas en los siguientes medios:

- El país

- El mundo

- $A B C$

- El correo gallego

- 20 minutos

- El confidencial

- El español

- RNE

- Onda cero

- Capital radio

- Antena 3

- La sexta

- Telemadrid

- Radiotelevisión del Principado de Asturias

- Radiotelevisión de Castilla y León

- Europa press.

Los profesionales que declinaron participar en el estudio señalaron distintos motivos. Los argumentos más expresados versaron sobre su no especialización en salud -por los motivos ya comentados-o por problemas de agenda y/o tiempo ya que se percibió en todos los casos una reducción de las plantillas de redactores y, por tanto, una acumulación del 
trabajo periodístico. Sin duda es un dato alarmante, teniendo en cuenta que es una especialidad de gran sensibilidad y calado entre la población.

No obstante se considera que la muestra es adecuada, puesto que quedan representados en el estudio todos los medios de comunicación. Cabe recordar las palabras de las investigadoras Rubio-Romero y Perlado-Lamo-de-Espinosa (2015), que indican que la muestra cualitativa no busca una representatividad estadística sino estructural, puesto que lo que pretende comprender son los distintos sentidos sociales y las relaciones con el fenómeno investigado, no la extrapolación de los datos al universo, como en el caso de la muestra cuantitativa.

La tabla 1 muestra los medios que toman parte en este trabajo.

Tabla 1. Medios participantes en el estudio

\begin{tabular}{|c|c|c|c|c|}
\hline Industria & Medio & $\begin{array}{l}\text { Código entrevis- } \\
\text { tado }\end{array}$ & N. medios & $\begin{array}{c}\% \text { respecto a la } \\
\text { muestra }\end{array}$ \\
\hline \multirow{7}{*}{ Prensa } & EL PAİS & E1 & \multirow{7}{*}{7} & \multirow{7}{*}{43,75} \\
\hline & ELEMUNDO & E2 & & \\
\hline & $\mathrm{ABC}$ & E3 & & \\
\hline & elCorreoGallego & E4 & & \\
\hline & 20 & E5 & & \\
\hline & El Confidencial & E6 & & \\
\hline & 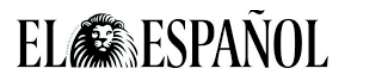 & E7 & & \\
\hline \multirow{3}{*}{ Radio } & roe & E8 & \multirow{3}{*}{3} & \multirow{3}{*}{18,75} \\
\hline & $\begin{array}{l}\text { ONDA } \\
\text { CERD }\end{array}$ & E9 & & \\
\hline & $\underbrace{\Theta}_{\text {capitalradio. }}$ & E10 & & \\
\hline \multirow{5}{*}{ Televisión } & 8 & E11 & \multirow{5}{*}{5} & \multirow{5}{*}{31,25} \\
\hline & 6 & E12 & & \\
\hline & theleMadrid & E13 & & \\
\hline & RTPa & E14 & & \\
\hline & rtvcyl.es & E15 & & \\
\hline Agencias & europa press & E16 & 1 & 6,25 \\
\hline \multicolumn{3}{|l|}{ Total medios } & 16 & 100 \\
\hline
\end{tabular}

\section{Resultados}

Se ordenan los resultados en función de los tres ejes temáticos de las entrevistas realizadas.

De los 16 medios de comunicación que participan en el estudio, sólo El mundo, Onda cero y RNE mantienen una sección especializada en salud, aunque en todos se difunden temas relacionados. Igualmente, la agencia de noticias Europa press mantiene un espacio temático denominado Infosalud. Se decidió incluir a las agencias (también a EFE en la muestra inicial) porque a través de sus portales actúan de medio de comunicación en abierto, ofreciendo informaciones generalistas y especializadas, en este caso sobre salud. 
En la mayor parte de los medios consultados, las secciones que aglutinan los contenidos de salud son Sociedad en primer lugar, y Ciencia en segundo término. Lo que no significa que en dichas secciones no haya especialistas en salud, como es el caso de El país o El correo gallego.
Para el $70 \%$ de los periodistas consultados, las nuevas tecnologías han promovido el aumento de contenidos sensacionalistas y morbosos sobre enfermedades

Todos los periodistas coincidieron en que es una espe-

cialidad que necesita preparación, formación y una especial sensibilidad, rigor y profesionalidad. Así lo apunta E10 (Capital radio):

"Vivimos un momento de sobreinformación, de exceso de datos y fuentes, muchas de ellas no especializadas y de origen dudoso, que exigen que el trabajo periodístico se vuelva más riguroso si cabe, especialmente cuando se centra en temas de ciencia y salud. Son áreas de especial sensibilidad para la opinión pública donde es imprescindible separar la información del ruido" (E10, Capital radio).

Recordemos que según el estudio Comunicar la ciencia: El perfil del periodista científico en España, el perfil de este profesional especializado en nuestro país es complejo y heterogéneo; sólo un tercio de los profesionales en activo cuenta con formación mixta en periodismo y ciencia, cuando se considera el perfil más idóneo. Existe la necesidad de crítica y análisis, no sólo de las fuentes sino de la propia información científica, que el periodista debe poder cuestionar (Cassany-Viladomat; Cortiñas-Rovira; Elduque-Busquets, 2018).

\subsection{Fuentes de consulta y fiabilidad de los datos}

En relación con las fuentes de consulta, en el estudio cualitativo se han localizado 15 referencias que marcan su procedencia. Los centros de investigación, instituciones sanitarias, asociaciones profesionales y gabinetes de comunicación fueron las fuentes más comentadas, seguidas de la Administración, asociaciones de pacientes, agencias especializadas, publicaciones científicas y universidades. También se mencionaron las entrevistas a fuentes directas pertenecientes a la red de contactos de cada profesional y los laboratorios. Entre los recursos menos utilizados, curiosamente se encuentran los medios internacionales, los medios especializados, los expertos en salud y las redes sociales.

Si nos centramos en estas dos últimas fuentes, los periodistas indican la necesidad de contar con expertos para verificar sus datos pero no siempre disponen de tiempo o recursos económicos para acceder a los mismos. Se vuelven a unir dos realidades:

- la rapidez en la producción informativa que domina los medios tras la digitalización;

- la reducción de los presupuestos de las redacciones para configurar las informaciones centradas en salud.

Las redes sociales no son fiables para los periodistas. De hecho, el $70 \%$ de los periodistas consultados indica que las nuevas tecnologías han promovido el ascenso de contenidos sensacionalistas y morbosos sobre todo en relación a enfermedades. E12 ( La sexta) comenta el peligro de publicar información contradictoria o de ofrecer falsas esperanzas. Por otro lado, un $68,4 \%$ de la muestra coincide en que tras la digitalización se deben tomar más precauciones con la información de salud y no utilizar las redes como fuente sino como canal de visualización y posicionamiento de sus piezas ya que ahora el periodista puede posicionar su producto y fomentar su repercusión. E2 (El mundo) recuerda que el comentario generado en redes puede dar ideas al periodista pero que hay que buscar siempre "más allá" para crear la noticia.

Como dato positivo, las redes sociales

"son muy útiles como puerta para acceder directamente a la fuente o a expertos" (E5, 20 minutos).

"Especialmente Twitter pone en contacto con muchísimos profesionales sanitarios; se ha generado una nueva manera de acceder a las fuentes" (E14, Radiotelevisión del Principado de Asturias).

E15 (Radiotelevisión de Castilla y León) lo resume así:

"La tecnología digital ha favorecido el tener acceso a mejores fuentes de divulgación científica. Ha ayudado a hacer el mundo más pequeño".

Las redes sociales permiten también un seguimiento continuo de la realidad y la comunicación directa y bidireccional con los seguidores:

"la nueva capacidad de interactuación del periodista no sólo con las fuentes sino con la audiencia” (E11, Antena 3).

"Al final la información sobre salud puede influir en la percepción sobre la propia salud de quienes lo consultan" (E16, Europa press).

Según los entrevistados, hay que tomar precauciones ante la desinformación y el exceso de noticias sin rigor ni profundidad, la revisión del origen de la fuente y su respaldo científico, el bulo y su propagación viral. También apuntan que: 
"hay que guardar y clasificar los enlaces de las fuentes ya que es más probable que se solicite argumentación que deba responderse de forma inmediata en el medio digital" (E8, RNE).

"La información de salud debe ser prudente, de servicio público y fiable", sentencia E9 (Onda cero).

Y es que "las noticias pueden ser usadas por los lectores como fuente de información para resolver sus propios problemas de salud y es muy importante ser especialmente claros" (E7, El español).

\subsection{Procesos de verificación}

Los procesos de verificación de la información especializada en salud pasan por diferentes estadios, que resumimos en el gráfico 1 , siempre según la opinión de los profesionales y en función del orden de citación.

Primero se demuestra la fiabilidad de las fuentes, se consulta a expertos y se contrastan los datos obtenidos. Luego se accede a la Administración o centro responsable de la información y, por último, se consultan aportaciones científicas incluidas en trabajos académicos o estudios de relevancia y se visitan webs de divulgación, siempre que estén referenciadas y sean recomendadas por la comunidad médico-científica. En relación con este último aspecto, E15 (Radiotelevisión de Castilla y León) sostiene:

"Pongo en cuarentena cualquier información publicada en una web que no tenga entre sus fuentes a sociedades científicas".

Y es que, como apunta E9 (Onda cero):

"Existe mucha desinformación que puede pasar por informa-

ción veraz y no lo es; eso exige contrastar y corregir".

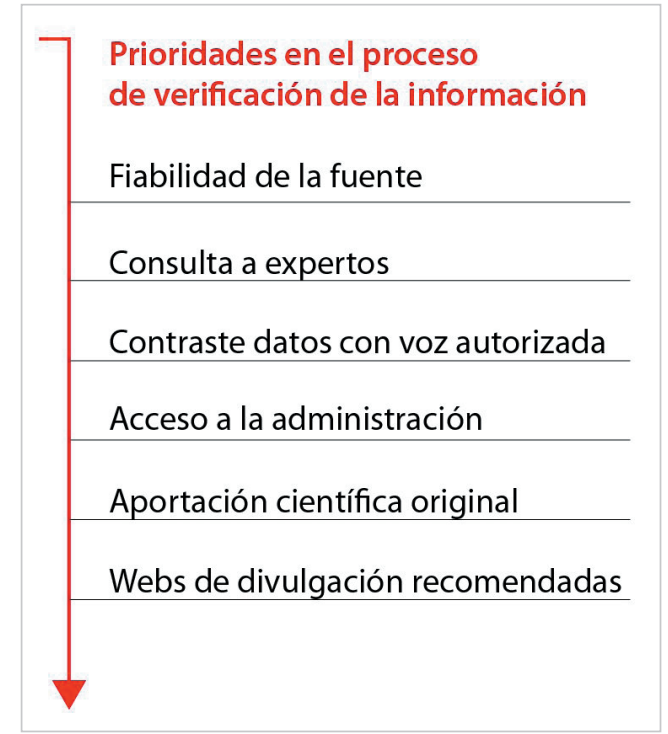

Gráfico 1. El proceso de verificación en la información de salud

E3 $(A B C)$ recuerda, en coincidencia con otros entrevistados, que el proceso de verificación se mantiene independientemente de la fuente y de la entrada de canales digitales:

"El mecanismo para un periodista es el mismo: comprobar la fiabilidad de la fuente y contrastar la información.

Ya se diga en rueda de prensa, nota de prensa, Twitter o Instagram".

Los periodistas coinciden en que la implementación de las nuevas tecnologías y el universo social media supone una oportunidad de reinvención y crecimiento, pero también una amenaza en cuanto a la pérdida de calidad del producto periodístico, la capacidad de actualización de los profesionales y la falta de tiempo provocada no sólo por la situación económica, también por la ampliación de las rutinas periodísticas.

Los profesionales citan una serie de nuevas funciones como por ejemplo:

- venta de temas en redes sociales;

- posicionamiento del trabajo realizado para "ganar en visibilidad" (E6, El confidencial);

- feedback con la audiencia;

- selección de cuentas de interés y el seguimiento de esos perfiles sociales;

- utilización de nuevas herramientas;

- fusión de las tecnologías para la creación de piezas multimedia en cualquier soporte.

\subsection{Desafíos profesionales}

El nuevo escenario de la información de salud provoca una serie de desafíos profesionales, según los periodistas entrevistados.

Todos coinciden en que no se debe sucumbir al trabajo fácil y que tienen que defender la profesión con seriedad. Se plantea el reto de combatir la celeridad actual de los medios de comunicación con un trabajo riguroso y contrastado. Hay que tomar conciencia de la necesidad de buscar la excelencia en el contenido, si cabe con más ahínco debido a la bidireccionalidad del medio digital y la capacidad de respuesta inmediata del receptor. Ante el exceso de información se debe proceder a una curación exhaustiva de los contenidos, discriminando datos y fuentes y, por tanto, mimando los procesos de verificación.

Estos esfuerzos protegerán al periodista y al medio de los bulos y las fake news, especialmente delicados en el periodismo de salud por la sensibilidad de estos contenidos y la afectación de la opinión pública. Igualmente, se debe educar a la sociedad en relación con las fuentes que debe considerar y el periodismo debe acoger de manera renovada la función informativa e incluso "la función divulgativa, para formar a la ciudadanía", como apunta E4 (El correo gallego). 
Otros desafíos profesionales derivados de la situación actual de los medios y el despliegue digital, vienen determinados por la necesidad de actualización de los periodistas ante este universo. Los entrevistados apuntan que ahora deben complementar el trabajo escrito con vídeo y podcast ante la integración de formatos, canales y medios. También deben utilizar herramientas de datos (data mining, data story, data science) para publicar piezas de investigación más profundas y depuradas y, como se ha apuntado, incluyen en sus rutinas el seguimiento de las redes sociales para posicionar sus temas, contactar con fuentes, interactuar con la audiencia, ampliar la información u observar la actualidad y los contenidos de interés.

Preocupa la falta de tiempo, la velocidad del cambio y la necesidad de formación. E14 (Radiotelevisión del Principado de Asturias) lo resume así:

"El desafío es la actualización".

Por último, los periodistas muestran una serie de preocupaciones en relación con la información de salud. E1 (El país) mantiene que

"se da difusión a informaciones sin base o mal enfocadas" y que "muchos medios del sector, por falta de financiación, bordean la línea del publirreportaje encubierto".

Ante la preponderancia de la imagen y la viralidad de las redes, existen

"demasiadas fotos explícitas e información sobre síntomas y tratamientos difíciles de discriminar", apunta E13 (Telemadrid).

Por su parte, E2 (El mundo) recuerda que en redes hay muchos temas "pinchables" que no están hechos con seriedad:

"es importante transmitir a los responsables que no se puede entrar en esa carrera".

En relación con ello, E8 (RNE) remarca el peligro de:

"favorecer el clickbait o ciberanzuelo en los medios, y no la información".

\section{Conclusiones}

En un contexto donde prima la digitalización y la generación de las redes sociales, los periodistas de salud se muestran preocupados por la falta de tiempo, el uso de recursos que fomentan el clickbait y la difusión de contenidos, en ocasiones cercanos al publirreportaje. Los usuarios valoran la presencia de estos canales de comunicación directos entre la comunidad y los expertos, pero observan con cierto escepticismo los posibles contenidos sensacionalistas y la desinformación. Los periodistas de salud usan como fuentes prioritarias los centros de investigación y las instituciones sanitarias.

La verificación resulta uno de los principales desafíos en la información de salud. En este proceso se contrasta la fiabilidad de las fuentes en primera instancia, seguido de la consulta a expertos y el contraste de los datos con voces autorizadas. Llama la atención que la consulta a expertos resulta ser una de las fuentes menos utilizadas en la generación del contenido por falta de tiempo o recursos, pero es la segunda más relevante en el proceso de verificación. Los profesionales buscan por tanto ofrecer información contrastada y de valor en aras de generar un contenido que, como se ha indicado en el trabajo, debe ser excelente por encontrar una amplia difusión en las redes sociales. Y por tratarse de una especialización muy sensible para la opinión pública, que en ocasiones acude a los medios buscando orientación sobre sus propios problemas.

Nuestros resultados sugieren otros desafíos derivados de la digitalización y relacionados con la celeridad e inmediatez en el ejercicio periodístico, como la curación de contenidos o el uso de herramientas de datos que puedan dar respuesta a la sobreabundancia de informa-

Sólo 4 de los 16 medios participantes en el estudio tienen una sección de salud ción. Los periodistas requieren de una formación y actualización continua en su especialización y en las aplicaciones relacionadas con su práctica profesional.

Por último, se recoge falta de inversión de los medios de comunicación en el área de salud. La mayoría no cuenta con esta especialización y su acontecer recae en la sección de Sociedad. Sólo 4 de los participantes en nuestra investigación la presentaban. Frente a la preocupación de los periodistas por hacer su trabajo con responsabilidad, la empresa informativa parece no dar valor a un contenido de alto impacto para la sociedad.

\section{Referencias}

APM (2017). Informe anual de la profesión periodística. Madrid: Asociación de la Prensa de Madrid. https://www.apmadrid.es/wp-content/uploads/2018/10/APM-Informe-2017_baja.pdf

Bauer, Martin V.; Howard, Susan; Romo-Ramos, Yulye J.; Massarani, Luisa; Amorim, Luis (2013). Global science journalism report: Working conditions \& practices, professional ethos and future expectations. London: SciDev. Net. ISBN: 978 1909528024

http://eprints.Ise.ac.uk/48051/1/Bauer_Global_science_journalism_2013.pdf 
Cassany-Viladomat, Roger; Cortiñas-Rovira, Sergi; Elduque-Busquets, Albert (2018). “Comunicar la ciencia: El perfil del periodista científico en España". Comunicar, v. XXVI, n. 55, pp. 9-18. https://doi.org/10.3916/C55-2018-01

Catalán-Matamoros, Daniel (2015). “Periodismo en salud: análisis de los públicos, formatos y efectos”. Panace@, v. 16, n. 42, pp. 217-224.

https://www.medtrad.org/panacea/IndiceGeneral/n42_tribuna-DCMatamoros.pdf

Cortiñas-Rovira, Sergi; Lazcano-Peña, Daniela; Pont-Sorribes, Carles (2015). "Periodistas científicos y efectos de la crisis sobre la información de ciencia: ¿hacia dónde va la profesión? Estudio del caso español”. Panace@, v. 16, n. 42, pp. 142-150. https://goo.gl/7soQQY

EHON (2017). Ehealth focus on. Documento de consenso sobre el estado de la información de salud en internet. Madrid: Asociación de Investigadores en eSalud (AIES).

http://laesalud.com/ehon/wp-content/uploads/2017/02/informe-ehon-01-salud-digital.pdf

González-Borjas, Antonia (2004). "Salud, información periodística especializada en alza”. Ámbitos, n. 12, pp. $301-310$. https://www.redalyc.org/articulo.oa?id=16801216

Infoperiodistas (2018). Infonómetro Salud 2018. Madrid: Infoperiodistas.

http://www.infoperiodistas.info/infonometro

Jiménez-Pernett, Jaime; García-Gutiérrez, José-Francisco; Martín-Jiménez, José-Luis; Bermúdez-Tamayo, Carmen (2007). "Tendencias en el uso de internet como fuente de información sobre salud". UOC papers, n. 4, pp. 44-50. http://www.redalyc.org/pdf/790/79000411.pdf

Marín-Torres, Viviana; Valverde-Aliaga, Justo; Sánchez-Miró, Ignacio; Sáenz-del-Castillo-Vicente, María-Isabel; Polentinos-Castro, Elena; Garrido-Barral, Araceli (2013). "Internet como fuente de información sobre salud en pacientes de atención primaria y su influencia en la relación médico-paciente”. Atención primaria, v. 45, n. 1, pp. 46-53.

https://doi.org/10.1016/j.aprim.2012.09.004

Meneses-Fernández, María-Dolores; Martín-Gutiérrez, Jorge (2015). “¿Tienen razón los investigadores al quejarse de la información periodística sobre ciencias? Experiencias con alumnos de Periodismo y científicos". Revista española de documentación científica, v. 38, n. 4, pp. 1-14.

https://doi.org/10.3989/redc.2015.4.1252

Pfizer (2010). El rol de internet en el proceso de consulta de información sobre salud. Madrid: Pfizer. https://www.pfizer.es/docs/pdf/noticias/Resultados_encuesta_Pfizer.pdf

Revuelta, Gema (2006). "Salud y medios de comunicación en España”. Gaceta sanitaria, v. 20, sup. 1, pp. $203-208$. http://www.gacetasanitaria.org/es-pdf-S0213911106715849

Revuelta, Gema; Minelli-de-Oliveira, Janaina (2008). “La salud y la biomedicina en la prensa diaria. Un análisis de diez años". Periodística: Revista académica, n. 11, pp. 55-67.

https://www.raco.cat/index.php/Periodistica/article/view/245697

Ronco-López, Milagros; Peñafiel, Carmen; Echegaray, Lázaro (2014). “El periodismo de salud en España. La información de salud en la prensa española (2000-2010): aproximación a los estudios documentales existentes". Documentación de las ciencias de la información, n. 37, pp. 267-304.

https://doi.org/10.5209/rev_DCIN.2014.v37.46827

Rubio-Romero, Juana; Perlado-Lamo-de-Espinosa, Marta (2015): “El fenómeno WhatsApp en el contexto de la comunicación personal: una aproximación a través de los jóvenes universitarios". Icono14, v. 13, n. 2, pp. 73-94.

https://icono14.net/ojs/index.php/icono14/article/view/818/526

Traver-Salcedo, Vicente; Fernández-Luque, Luis (2011). El ePaciente y las redes sociales. Valencia: Publidisa. ISBN: 978 8469441107

https://ec.europa.eu/eip/ageing/sites/eipaha/files/results_attachments/libro_el_epaciente_y_las_redes_sociales.pdf

Ufarte-Ruiz, María-José (2012). “La situación laboral del periodista como factor condicionante de la calidad informativa: con precariedad no hay calidad". En: Congreso Internacional Latina de Comunicación Social, Universidad de La Laguna, Tenerife.

http://www.revistalatinacs.org/12SLCS/2012_actas/037_Ufarte.pdf

Waisbord, Silvio; Coe, Gloria (2002). "Comunicación, periodismo, salud y desafíos para el nuevo milenio". Razón y palabra, n. 26.

http://www.razonypalabra.org.mx/anteriores/n26/swaisbord.html

Wellcomm (2012). Perspectivas de la comunicación. Madrid: Wellcomm.

https://well-comm.es/2012/01/26/perspectivas-de-la-comunicacion-2012 